\title{
Tactile exploration of visual reinforcement following operant responding by 12 - to 24-month-old infants
}

\author{
CHRISTINA FANTAUZZO, CAROL STECIK, and RICHARD DENI \\ Rider College, Lawrenceville, New Jersey
}

\begin{abstract}
Two experiments studied the interaction of operant responding for visual reinforcement (color slides) and tactile exploratory behavior directed toward that reinforcement. Experiment 1 attempted to quantify the relationship between operant performance and tactile exploration of the reinforcement produced. In an effort to influence exploratory tendencies, certain infants were given a tactile "priming" treatment of prior play exposure to the materials used as visual reinforcers. No differences in exploratory tendencies were found as a function of this prior exposure. However, in those infants who did not play before operant learning, a significant correlation was found between operant response frequency and total amount of tactile exploration of the visual images used as reinforcement. Visual reinforcers did elicit tactile responses. In Experiment 2, infants were again given the opportunity to touch visual reinforcement stimuli, this time during two different types of operant contingencies: buttonpress and "step on floor pad." Those reinforcing stimuli activated by the floor-pad response were explored (touched) significantly more, indicating that the tendency to explore reinforcers depended upon the manner in which they were produced. These data were discussed by relating certain types of exploration as "adjunctive" behavior in an operant contingency.
\end{abstract}

The classic view of the role of exploration in cognitive development during infancy describes a general process whereby the child is drawn to novel environments and novel objects by the fortunate consequence that a new learning opportunity will result (Ross, Rheingold, \& Eckerman, 1972). Consistent with this view has been the frequent finding that, as infants became increasingly familiar with specific reinforcing consequences, they showed less inclination to be conditioned by them (Siqueland \& DeLucia, 1969). Thus, exploration of a novel event has the potential to stimulate learning, but also plays a role in the process whereby infants become bored with familiar contingencies. Whether infants are bored with the contingency or the reinforcement is unknown. Direct measures of exploratory behavior, separate from operant behavior, would provide data that could begin to answer such a question.

For the most part, studies of infant operant behavior have presented reinforcement to specific sensory channels: Infants were given pictures to look at, sounds to hear, etc. The "consumption" of the reinforcement in those situations was presumably the looking and listening on the part of the infants. However, in many studies, this "consummatory" behavior was not directly measured, and indications of the perception of a contingency by infants were related to transitions in operant behavior between baseline and conditioning phases. Between-groups comparisons have also been used as evidence for a rise

The authors' mailing address is: Department of Psychology, Rider College, Lawrenceville, NJ 08648. or fall in the perception of a contingency, as in the case of the Finkelstein and Ramey (1977) study, in which infants differed in whether they received contingent or noncontingent stimulation during operant performance sessions.

The experiments reported were sought to add a new dimension to the question of whether the opportunity to explore and become familiar with reinforcement affects operant behavior. Specifically, infants were given tactile exploratory opportunities with visual reinforcing stimuli. The general assumption was that when infants are given extra opportunities to "consume" reinforcing stimuli, their operant behavior would be differentially affected. In Experiment 1, prior to performing a manual operant contingency, infants were given tactile play experience with picture stimuli similar to what was later used as visual reinforcement. The study sought to determine what effect "priming" the exploratory tendency would have on further tactile exploration operant reinforcers. In Experiment 2, the operant behavior of infants was either matched or mismatched to a tactile exploratory opportunity. The study tested whether exploration of reinforcing stimuli through one sensory channel (tactile) affected performance of an operant contingency designed for a different channel (visual).

\section{GENERAL METHOD}

\section{Subjects}

The subjects were infant boys and girls obtained through newspaper birthday and birth announcements. Families participated on a volunteer basis. Only infants capable of walking independently were tested. 


\begin{abstract}
Apparatus and Test Environment
The operant test room was a rectangular space, $3 \times 2 \mathrm{~m}$. Two operant "stations" were present in the test room, one on each side of a low partition. Two Kodak Carousel projectors were located behind this partition and set to display color slides of the "Sesame Street" characters on two separate rear-projection screens (image size of approximately $40 \times 15 \mathrm{~cm}$ ). Each screen was covered by a wooden rectangular "hood" constructed to provide a $40 \times 15 \mathrm{~cm}$ aperture through which the subjects could reach to touch the screen. A bank of photocells monitored touches directed toward the screen. An operant response button was located to the side of each screen. An additional response device was available at each station in the form of a $60 \times 60 \mathrm{~cm}$ weight-sensitive switch pad located on the floor in front of each screen. The three input devices (buttons, switch pads, and photocells), projector lamps, and carousel advance controls, were interfaced with a TRS- 80 Model 4 computer through a Med Associates LVB interface. The computer controlled all contingencies and data collection in real time.
\end{abstract}

\section{EXPERIMENT 1}

Tactile Exploration of a Visual Reinforcement Following Visual and Tactile Play by 12- to 24-Month-Old Infants

\section{Method}

The subjects were 20 infants, 10 boys and 10 girls, ranging in age from 12 to 24 months. Prior to being tested in the operant room, the subjects were taken to a small play room. This play room contained a small table and two chairs. Play materials consisted of 10 "cards," each showing a Sesame Street Muppet character, and 5 plastic Muppet finger puppets. The procedure was divided into two phases.

In Phase 1, the subjects were assigned randomly to one of two conditions: play and no-play. Parents and infants were escorted to the play room and seated on one side of a small table. Parents held the infants in their laps, and the experimenter was seated at the opposite side of the table. In the play condition, the experimenter presented the Muppet cards and finger puppets to the child, who explored them visually and tactually. The stimuli were presented separately in an arbitrary sequence. Exposure to each stimulus lasted until the subject seemed satisfied (between 10 and $20 \mathrm{sec}$ ). In the no-play condition, the experimenter talked quietly to the parent and infant for a comparable period (between 4 and $5 \mathrm{~min}$ ).

In Phase 2, both groups were tested on the same operant task. Each parent and infant were escorted into the operant test room. In this experiment, only the left operant station was operative, and only the response button and left-side photocells were used as input devices. The parent was seated in the center of the room, and the infant was free to walk. The parent was instructed to show the infant the location of the response button and screen. During the 6-min test esssion, the subjects received $5 \mathrm{sec}$ of exposure to color slides of Sesame Street characters contingent upon each operant buttonpress. The schedule of reinforcement was a fixed-ratio one. Responses made during slide exposures were ignored, and only the number of reinforcements earned was recorded as the measure of operant behavior. In addition, two exploratory measures were also recorded: (1) the frequency and (2) the duration of breaks of the photobeams. These measures were recorded during and between reinforcement exposures.

\section{Results and Discussion}

Two types of raw data were obtained: (1) the total number of operant buttonpresses that were reinforced, and (2) the frequency and duration of exploratory touches directed toward the visual reinforcement (or the location where reinforcement was presented). Two average exploration scores were derived for each subject: the average exploratory movement per reinforcement when the color slide was actually on, and the average exploratory movement per reinforcement when the slide was not present.
These two scores served as measures of exploratory behavior during and between individual visual reinforcement presentations.

Relationship between operant behavior and tactile exploration. Three product-moment correlations were done to relate the total number of reinforced buttonpresses and the total duration of exploration during reinforcement. These correlations were: overall (20 pairs of scores), for play subjects only (10 pairs), and for no-play subjects only (10 pairs). The presence of a strong correlation between tactile exploration and operant performance was presumed to support the notion of consummatory behavior in more than just the "target" (visual) channel. There was a significant overall correlation between the two measures $[\mathrm{r}(18)=.54, \mathrm{p}<.05]$. The correlation for the play subjects only was not significant $[\mathrm{r}(8)=.45, \mathrm{p}>.05]$. However, significance was reached for the no-play subject $[r(8)=.76, p<.05]$. Clearly, when infants did not get prior tactile play experience with stimuli like those to be used as visual reinforcement (did not experience " "priming"), their subsequent operant performance for visual reinforcement was synchronized with their tactile exploratory behavior. These findings support the view that infants were consuming reinforcement using more than just the target channel, and that prior activation of extra channels may relate to how the operant contingency is performed. The data also suggest that exploratory behavior was an adjunctive response patterned by the operant contingency.

The specific test of the "priming" hypothesis came in the form of analyses of variance on both the operant and exploration data. Two independent variables were common to all analyses: sex of subject and prior play/exploration experience during Phase 1 (play, no-play).

Operant responding. No significant differences in operant buttonpressing were found as a function of either the sex of subject or prior play/exploration with the reinforcing stimuli. Boys made slightly more reinforced operant responses (mean $=37.3, \mathrm{SD}=4.1$ ) than girls (mean $=31.5$, SD $=8.2$ ). The summary of operant performance in the play condition (mean $=34.5, \mathrm{SD}=7.9$ ) was identical to that in the no-play condition (mean = 34.3, SD = 5.4).

Exploratory behavior - sex and play conditions. No significant differences in tactile exploratory behavior were found as a function of either the sex of subject or prior play/exploration with the reinforcing stimuli. Boys (mean $=0.7, \mathrm{SD}=0.7$ ) and girls (mean $=0.7, \mathrm{SD}=0.6$ ) made the same duration of exploratory responses per reinforcement. Exploratory responses made in the play condition (mean $=0.8, \mathrm{SD}=0.8$ ) were slightly longer than those made in the no-play condition (mean $=0.6, \mathrm{SD}=$ 0.6 ).

Exploratory behavior-during vs. between reinforcement. The subjects explored significantly more during (mean $=0.9, \mathrm{SD}=0.8$ ) than between (mean $=0.5, \mathrm{SD}$ $=0.5$ ) individual visual reinforcements $[F(1,16)=5.20$, $\mathrm{p}<.05]$. This finding suggests that tactile exploration 
by infants can serve as a separate indicator of perception of a contingency (separate from operant performance per se). The tactile exploratory behavior measured was more often specifically directed toward the visual reinforcement and not merely the place where it was presented.

Taken together, these findings cannot support the "priming"' hypothesis as originally presented. However, there were clear indications that operant responding and the opportunity to explore the visual reinforcement were functionally linked. Experiment 2 was an attempt to study the specific nature of that linkage by setting up conditions in which operant behavior was either matched or mismatched with the opportunity for tactile exploration of visual reinforcement.

\section{EXPERIMENT 2 \\ Effects of Type of Contingency on Tactile Exploration of Visual Reinforcement in 12- to 20-Month-Old Infants}

\section{Method}

Six boys and six girls, ranging in age from 12 to 20 months, were tested. In this experiment, only the operant test room was used. Both operant stations were used, and the floor-mounted switch pads provided an additional input device.

All preparations prior to the start of operant testing were the same as those in Experiment 1. Each test session lasted $10 \mathrm{~min}$. During the session, the subjects were free to move between the two response stations to operate the slide projectors. A switch closure at either station resulted in a 5 -sec slide exposure on the corresponding screen. During each slide exposure, a recording was made of the amount of time the corresponding photo beams were broken by exploratory touches. The right response station was activated by a keypress, whereas the left response station was activated when a subject simply stood on the floor pad in front of the left screen.

Two dependent measures were recorded: the cumulative duration of photobeam breaks during slide presentations and the frequency of reinforced operant responses at each station.

\section{Results and Discussion}

The duration of beam breaks was converted to a ratio measure to equalize differences in operant behavior at the two response stations. The resulting score was derived by dividing the total duration of beam breaks by the total frequency of reinforced operant responses. A separate beam-break score was obtained for performance at each station. Operant response data and beam-break scores were tested for group differences using separate two-factor mixed analyses of variance. The between-subjects factor in the analyses was the sex of subject. The within-subjects factor was response station (button vs. floor pad).

Operant responding. The analysis of operant response data revealed a single significant effect $[\mathrm{F}(1,10)=5.18$, $\mathrm{p}<.05]$. A higher response total was obtained at the floor-pad response station than at the buttonpress station. The means and standard deviations for this effect are shown in the top panel of Table 1. Apparently, infants preferred to produce their visual reinforcement by gross motor means rather than by pressing a button with the finger. However, all subjects made buttonpress responses,
Table 1

Means and Standard Deviations for Operant Response Performance and Duration of Manual Exploration at the Location Where Slide Reinforcement was Presented as a Function of the Type of Operant Contingency

\begin{tabular}{|c|c|c|}
\hline Response Task & Mean & SD \\
\hline \multicolumn{3}{|c|}{ Frequency of Reinforced Operant Responses } \\
\hline $\begin{array}{l}\text { Response Button } \\
\text { Floor Pad }\end{array}$ & $\begin{array}{l}17.6 \\
29.5\end{array}$ & $\begin{array}{l}10.7 \\
15.1\end{array}$ \\
\hline \multicolumn{3}{|c|}{ Average Duration (in seconds) of Beam Breaks per Reinforcement } \\
\hline $\begin{array}{l}\text { Response Button } \\
\text { Floor Pad }\end{array}$ & $\begin{array}{l}0.3 \\
0.9\end{array}$ & $\begin{array}{l}0.4 \\
0.7\end{array}$ \\
\hline
\end{tabular}

and, on average, half of the reinforcement was obtained through that contingency.

Exploratory behavior. The analysis of duration of beam breaks per reinforcement revealed the same significant effect $[F(1,10)=12.25, p<.001]$. More time was spent reaching toward the screen per reinforcement at the floor-pad response station than at the buttonpress station. The means and standard deviations for this effect are shown in the lower portion of Table 1 . It must be remembered that these scores were ratios corrected in terms of absolute differences in button versus pad preference. The clear implication of these data is that infants who do not use manual operants to produce visual reinforcement are more likely to show tactile exploration of those consequences.

\section{GENERAL DISCUSSION}

The results of these two experiments are particularly useful in determining the direction for future studies along this path. First, the Experiment 1 data showed that operant performance and exploration of the reinforcer were correlated. However, a direct test of how one might gain experimental control over this relationship was not clearly verified, since the "priming" hypothesis was not proved. On the other hand, the Experiment 2 data successfully demonstrated that operant-exploratory linkages can be obtained when the type of contingency is the variable under study. The particular problem left by the results of Experiment 2 was the direction of the difference. It would have been more clear had the exploratory tendency been more pronounced when the consequence was produced by manual means. Nevertheless, the data encourage the conceptualization of certain types of exploratory behavior as "adjunctive" to the operant contingencies in effect when they occur. For example, one interpretation of the Experiment 2 data suggests that a contingency that does not involve manipulation produces more "adjunctive" tactile exploration than one that requires hand and finger movement as part of the operant behavior.

There are specific benefits to studying exploratory behavior as an "adjunctive" correlate of a learned contingency. According to Bijou (1980), exploratory tendencies usually develop under the influence of nonexploratory experience, either prior to the opportunity to explore or coincident with it. Play experiences are a classic example, although there has been considerable disagreement on the closeness of the functional relationship of play and exploration. The conceptualization of certain types of exploration as "adjunctive" responses would bypass this confusion in the functional definition of exploratory behavior. Subtle evidence for the adjunctive response definition of exploration has been provided by Antonitis (1978), who reported that although children stopped barpressing when they learned that they were not causing free reinforcements, they also stopped exploration directed at those free rein- 
forcements. Data of this type indicate the need to work toward making a stronger case for that definition.

\section{REFERENCES}

ANTonitis, J. J. (1978). Motivating effects of free auditory stimuli on the bar pressing behavior of preschool children in a real-life situation. Journal of Genetic Psychology, 133, 79-80.

BiJou, A. Q. (1980). Exploratory behavior in infants and animals: A behavior analysis. Psychological Record, 30, 483-495.
Finkelstein, N. W. \& RAMEY, C. T. (1977). Learning to control the environment in infancy. Child Development, 48, 806-819.

Ross, H. S., RHEInGold, H. L., \& ECKeRMan, C. O. (1972). Approach and exploration of a novel alternative by 12 -month-old infants. Journal of Experimental Child Psychology, 13, 85-93.

Siqueland, E. R., \& Delucia, C. A. (1969). Visual reinforcement of nonnutritive sucking in human infants. Science, 165, 1144-1146.

(Manuscript received for publication January 7, 1985.) 